\title{
optica
}

\section{Polarization-assisted amplitude gating as a route to tunable, high-contrast attosecond pulses: supplementary material}

\author{
Henry Timmers, ${ }^{1,+,}{ }^{*}$ Mazyar Sabbar, ${ }^{1, \dagger}$ Johannes Hellwagner, ${ }^{1}$ YUkI \\ Kobayashi, ${ }^{1}$ Daniel M. Neumark, $1,2, *$ and Stephen R. Leone $1,2,3, *$ \\ ${ }^{1}$ Department of Chemistry, University of California, Berkeley, CA 94720, USA \\ ${ }^{2}$ Chemical Science Division, Lawrence Berkeley National Laboratory, Berkeley, CA 94720, USA \\ ${ }^{3}$ Department of Physics, University of California, Berkeley, CA 94720, USA \\ *Corresponding author: htimmers@berkeley.edu,srl@berkeley.edu,dneumark@berkeley.edu \\ ${ }^{\dagger}$ These authors contributed equally to this work. \\ Published 28 June 2016
}

This document provides supplementary information to "Polarization-assisted amplitude gating as a route to tunable, high-contrast attosecond pulses," http://dx.doi.org/10.1364/optica.3.000707. We provide additional detail concerning the PASSAGE experimental set-up, a discussion on the attosecond streaking technique, an explanation of the FROG-CRAB reconstruction algorithm, and an overview of the pulse energy measurement. Finally, a detailed explanation of the strong field approximation simulation is provided to simulate high harmonic spectra using the PASSAGE technique. (C) 2016 Optical Society of America

http://dx.doi.org/10.1364/optica.3.000707.s001

\section{EXPERIMENTAL SET-UP}

Femtosecond pulses are generated from a CEP-stable oscillator (Femtolasers GmbH, Rainbow CEP 3 Oscillator) and amplified (Femtolasers $\mathrm{GmbH}$, Femtopower Amplifier) to a pulse energy of $1.8 \mathrm{~mJ}$ at $1 \mathrm{kHz}$ and a temporal duration of $25 \mathrm{fs}$. The CEP is stabilized to $\sim 100 \mathrm{mrad}$ after the amplifier. The CEP-stable pulses are then compressed using the combination of a Ne-filled stretched hollow-core fiber (Few-Cycle Inc.) to broaden the pulse spectrum and a set of broadband chirped mirrors to compress the femtosecond pulses to $5 \mathrm{fs}$ durations at a pulse energy of 0.8 $\mathrm{mJ}$.

The experimental set-up for isolating and characterizing attosecond pulses generated via PASSAGE is shown in Fig. S1. The few-cycle pulses are divided into a pump and probe path with a broadband 70:30 beamsplitter. The pump beam is sent through the PASSAGE optics described in the paper to shape the polarization of the pulse and then focused with a $65 \mathrm{~cm}$ lens into a $2 \mathrm{~mm}$ gas cell. A broadband HHG spectrum is generated by filling the gas cell with either 30 torr of Ar gas or 150 torr of Ne gas for optimal attosecond pulse production. The driving NIR field is then filtered out using either a $200 \mathrm{~nm} \mathrm{Al}$ filter or multi-channel plate (MCP) filter [1] + $200 \mathrm{~nm} \mathrm{Zr}$ filter to isolate attosecond pulses below or above $72 \mathrm{eV}$, respectively.

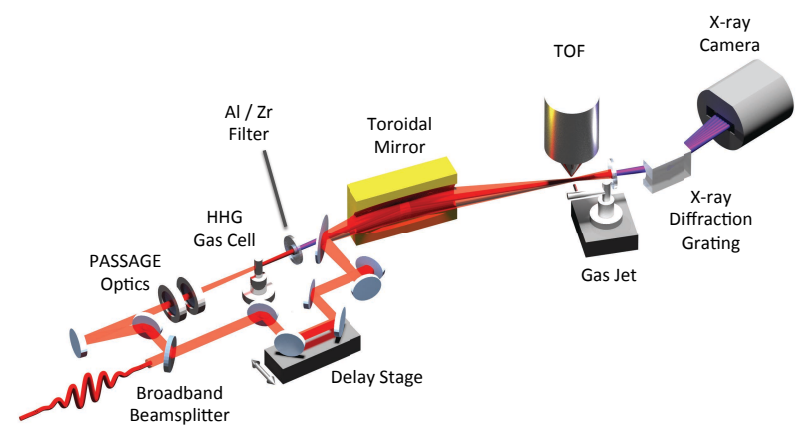

Fig. S1. The experimental apparatus used to isolate and characterize the attosecond pulses generated via PASSAGE.

The attosecond pulses are characterized using attosecond streaking spectroscopy. For this technique, the attosecond and NIR pulses are focused by a toroidal mirror into an interaction region containing an effusive gas jet of $\mathrm{Ne}$. The attosecond pulses coherently ionize the Ne ensemble, launching a photoelectron wavepacket. The time-delayed NIR probing pulse is used to 
streak the energy distribution of the photoelectron wavepacket. The streaked photoelectrons are measured with a time-of-flight spectrometer and the signal is digitized using a 250 ps time-todigital converter. The delay-dependent photoelectron spectrum yields a streaking spectrogram that is characterized by a unique attosecond-pump and NIR-probe field. To reconstruct the temporal profiles of the pump and probe pulses, an iterative reconstruction algorithm is implemented (discussed in the following section).

Finally, the spectrum of the attosecond pulse is measured with a homebuilt X-ray spectrometer. The spectrometer consists of a concave, aberration-corrected diffraction grating and an $\mathrm{x}$ ray sensitive CCD camera. The spectrometer is calibrated using ionic and neutral absorption features in $\mathrm{Xe}(55-65 \mathrm{eV})$ along with the $\mathrm{Al}$ absorption edge at $72.6 \mathrm{eV}$. The resolution of the $\mathrm{x}$-ray camera is found to be around $100 \mathrm{meV}$ at $55 \mathrm{eV}$.

\section{FROG-CRAB RECONSTRUCTION ALGORITHM}

A variation of the FROG-CRAB algorithm is used to reconstruct the attosecond pulse duration from the experimental streaking spectrogram. The FROG-CRAB algorithm is based on the LeastSquares Generalized Projection Algorithm (LSGPA) developed by Gagnon et al. [2] and uses an iterative approach to minimize the error between the experimental spectrogram $\left(S_{\text {exp }}\right)$ and the reconstructed spectrogram $\left(S_{\text {recon }}\right)$. This reconstructed spectrogram is uniquely determined by a pulse vector (given by the temporal field of the attosecond pulse) and a gate vector (given by the delay-dependent phase modulation of the photoelectron wavepacket induced by the NIR streaking field) pair which are retrieved at each iteration of the algorithm. The error of the FROG-CRAB reconstruction is defined by

$$
\sigma=\sqrt{\frac{2 \sum_{i, j}\left[\left(S_{\text {recon }}^{i, j}\right)^{2}-\left(S_{\text {exp }}^{i, j}\right)^{2}\right]}{\sum_{i, j}\left(S_{\text {recon }}^{i, j}\right)^{2}+\sum_{i, j}\left(S_{\text {exp }}^{i, j}\right)^{2}}},
$$

where $i$ and $j$ represent indices along the energy and delay axis, respectively. In addition to the FROG-CRAB error, an uncertainty in the reconstructed attosecond pulse duration can also be obtained through the LSGPA algorithm [3]. Within the algorithm, a signal matrix is defined using the expression $S_{i j}=P_{i} G_{i+L(j-1)}$, where $L$ is an integer representing the time samples per delay step. This signal matrix is a measure of the reconstructed pulse at each delay point in the spectrogram. The matrix therefore provides a statistical distribution of reconstructed pulses that can be used to quantify the degree of uncertainty in the reconstructed pulse duration.

The experimental streaking spectrogram for an attosecond pulse isolated in Ne via $45^{\circ}$ PASSAGE is shown in Fig. S2 (a) [Fig. 2 (a) in the paper]. The corresponding reconstructed spectrogram, XUV spectrum, and attosecond temporal profile are shown in S2 (b), (c), and (d), respectively. The FROG-CRAB error for this reconstruction was found to be $\sigma=0.2 \%$.

The experimental streaking spectrogram for an attosecond pulse isolated in Ar via $45^{\circ}$ PASSAGE is shown in Fig. S3 (a) [Fig. 2 (c) in the paper]. The corresponding reconstructed spectrogram, XUV spectrum, and attosecond temporal profile are shown in S3 (b), (c), and (d), respectively. The FROG-CRAB error for this reconstruction was found to be $\sigma=0.13 \%$.

\section{MEASUREMENT OF ATTOSECOND PULSE ENERGY}

A calibrated XUV photodiode (AXUV63HS1, Opto Diode Corp.) is used to measure the pulse energy of the attosecond pulses (a)
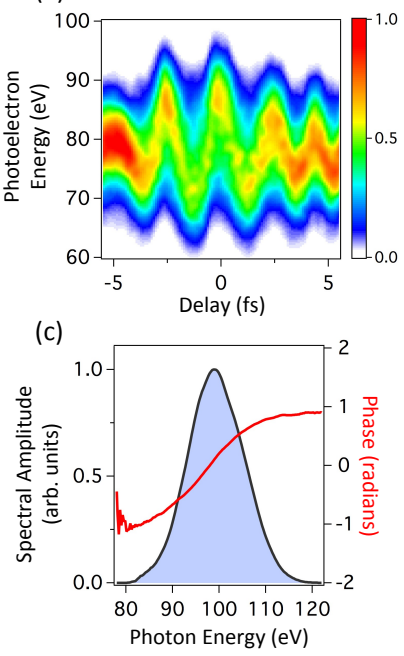

(b)

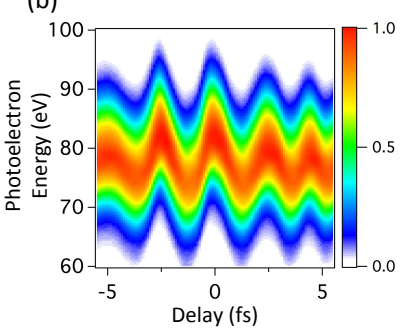

(d)

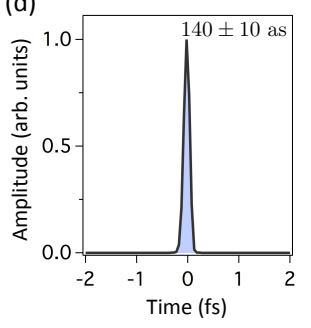

Fig. S2. (a) The experimental [Fig. 2 (c) in paper] and (b) reconstructed streaking spectrogram for attosecond pulses isolated via $45^{\circ}$ PASSAGE in Ne. The LSGPA algorithm reconstructs the XUV spectrum and attosecond temporal profile shown in (c) and (d) respectively. The corresponding FROGCRAB error is $\sigma=0.2 \%$.
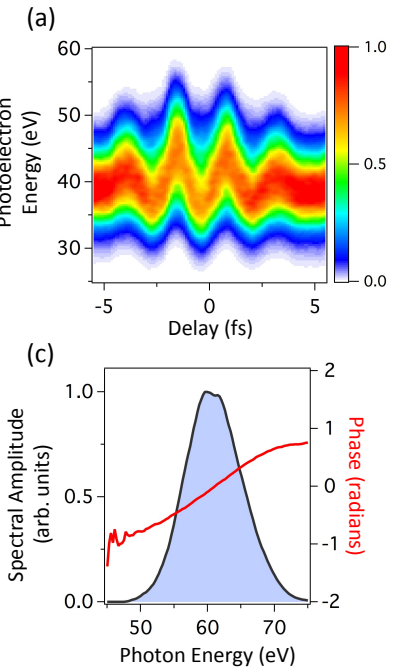

(b)

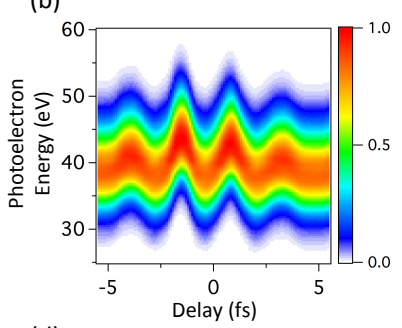

(d)

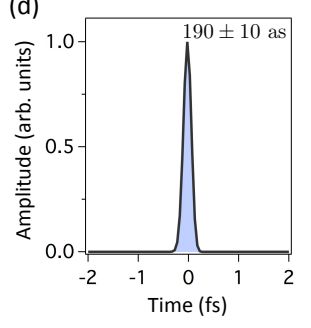

Fig. S3. (a) The experimental [Fig. 2 (d) in paper] and (b) reconstructed streaking spectrogram for attosecond pulses isolated via $45^{\circ}$ PASSAGE in Ar. The LSGPA algorithm reconstructs the XUV spectrum and attosecond temporal profile shown in (c) and (d) respectively. The corresponding FROGCRAB error is $\sigma=0.13 \%$. 


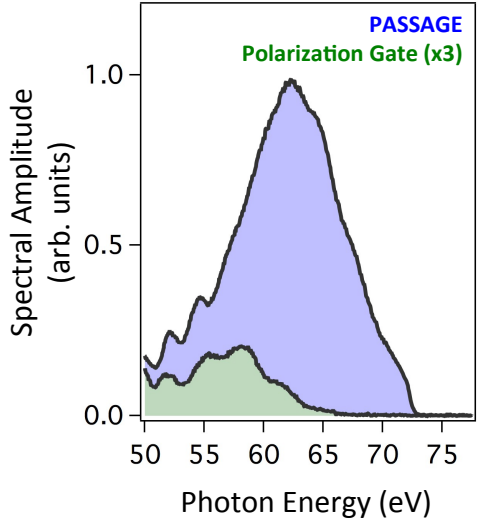

Fig. S4. A comparison between a SAP isolated in Ar gas using PASSAGE (blue-shaded curve) and polarization gating (green-shaded curved, multiplied by a factor of three). The implementation of the full polarization gate reduces the cut-off spectrum above the Ar Cooper minimum by a factor of $\sim 20$.

isolated via PASSAGE in an Ar HHG medium. The photodiode is mounted on a linear vacuum feedthrough placed directly after the toroidal mirror. To measure the pulse energy in the HHG generation cell, we take into account the measured transmission of the $\mathrm{Al}$ filter (30\% transmission) and the calculated reflection off the Au-coated toroidal mirror (60\% transmission) [4] to arrive at a HHG source energy of 0.73 nJ. However, this value corresponds to the integrated pulse energy of the entire HHG spectrum. Our X-ray spectrometer has a working range of 50-200 $\mathrm{eV}$ and therefore cannot be used to disentangle the pulse energy contribution above the Cooper minimum, corresponding to the SAP. Therefore, a photoelectron spectrum is acquired in He to measure a contribution of $75 \%$ for the HHG spectrum above the Cooper minimum, corresponding to a SAP pulse energy of $\sim 0.6$ $\mathrm{nJ}$. This agrees well with the value of $0.56 \mathrm{~nJ}$ estimated using the
X-ray spectrometer.

To isolate attosecond pulses in Ne via PASSAGE, an additional MCP filter placed in front of the $\mathrm{Zr}$ filter is required to prevent laser-induced damage of the $\mathrm{Zr}$ filter. This leads to a $\sim 80 \%$ drop in the XUV pulse energy. As a result, the pulse energy is too low to measure with the XUV photodiode. Instead, given the sound agreement between the XUV photodiode and the $X$-ray spectrometer measurement techniques with pulses isolated in Ar, we can use the X-ray spectrometer to estimate a Ne pulse energy of $0.2 \mathrm{~nJ}$.

\section{COMPARISON BETWEEN PASSAGE AND POLARIZA- TION GATING}

Polarization gating is a good technique for isolating low energy plateau harmonics. However, due to the substantial reduction in peak intensity, polarization gating cannot be used to isolate the cut-off portion of the HHG spectrum. This can be easily seen in Fig. S4. When PASSAGE is used to generate a SAP using Ar as a driving medium (blue-shaded curve), a broad cut-off spectrum is obtained stretching from 50 to $72.6 \mathrm{eV}$. However, when the full polarization gate is turned on (green-shaded curve, multiplied by a factor of three), the counts within the cut-off are substantially reduced (by a factor of 20) and what remains of the cut-off no longer extends to $72.6 \mathrm{eV}$. The situation is even worse using $\mathrm{Ne}$ as a driving medium. While SAP spectra extending from 80-120 eV can easily be obtained using PASSAGE, the counts diminish to zero when the full polarization gate is turned on.

\section{NUMERICAL SIMULATION OF HHG SPECTRA}

The Lewenstein model $[5,6]$ is used to simulate the HHG spectrum generated in Ne by the time-dependent, elliptically polarized field. Within the Lewenstein model, the strong field approximation is invoked to express the time-dependent atomic dipole in the strong laser field, $\vec{d}(t)$, as

$$
\vec{d}(t)=-i \int_{0}^{\infty} d \tau\left(\frac{\pi}{\epsilon+i \tau / 2}\right)^{3 / 2}\left\{\vec{E}(t) \cdot \vec{D}\left[\vec{p}_{s}(t, \tau)-\vec{A}(t-\tau)\right]\right\} e^{-i S_{s}(t, \tau)} \vec{D}^{*}\left[\vec{p}_{s}(t, \tau)-\vec{A}(t)\right]|a(t)|^{2}+\text { c.c. }
$$

where $\tau=t_{r}-t_{i}$ is the time delay between ionization $\left(t_{i}\right)$ and recollision $\left(t_{r}\right)$ of the electronic wavepacket, $\epsilon$ is a small constant to remove the singularity at $\tau=0, \vec{E}(t)$ is the time-dependent, elliptically polarized electric field of the driving laser pulse, $\vec{A}(t)=-\int_{-\infty}^{t} d t \vec{E}(t)$ is the corresponding vector potential, $\vec{D}(\vec{p})$ is the dipole matrix element between the ground atomic state and a continuum state with electron momentum $\vec{p}$, and $|a(t)|^{2}$ is the time-dependent ground state amplitude calculated using ADK theory. Invoking the saddle-point method, the main contribution to the time-dependent dipole is an electron trajectory with a stationary momentum and action given by,

$$
\vec{p}_{S}(t, \tau)=\int_{0}^{\tau} d t^{\prime \prime} \vec{A}\left(t^{\prime \prime}\right) / \tau
$$

and

$$
S_{s}(t, \tau)=\int_{0}^{\tau} d t^{\prime \prime}\left\{\frac{\left[\vec{p}_{s}(t, \tau)-A\left(t^{\prime \prime}\right)\right]^{2}}{2}+I_{p}\right\},
$$

respectively, where $I_{p}$ is the ionization potential of Ne. In addition, phase-matching plays an important role in isolating the short trajectories for the generation of single attosecond pulses [7]. Therefore, we take into account macroscopic propagation by solving the on-axis wave propagation equation [8]

$$
\frac{\partial E_{x}(\omega, z)}{\partial z}=-\frac{i}{2} \omega c \mu_{0} P_{x}^{n l}(\omega, z)
$$

where $E_{x}(\omega, z)$ is the electric field spectrum of the attosecond pulse polarized along the $x$-direction, $z$ is the direction of propagation, $\omega$ is the frequency of the attosecond field, $c$ is the speed of light in vacuum, $\mu_{0}$ is the permeability of free space, $P_{x}^{n l}(\omega, z)=n_{0} d_{x}(\omega)$ is the non-linear polarization in the $x$ direction, $n_{o}$ is the atomic density of $\mathrm{Ne}$ in the interaction region, and $d_{x}(\omega)$ is the $x$-component of the Fourier transform of the time-dependent dipole in Eq. 2.

To model the spectrum of an attosecond pulse generated with linear polarization vs. PASSAGE, the single-atom response given in Eq. 2 is numerically solved and the build-up of harmonic intensity is propagated using Eq. 5. Since a $2 \mathrm{~mm}$ gas cell is used in the experiment, we propagate the equation for $2 \mathrm{~mm}$ 
with a step size of $20 \mu \mathrm{m}$ from $z_{r}-1 \mathrm{~mm}$ to $z_{r}+1 \mathrm{~mm}$, where $z_{r}=2.27 \mathrm{~cm}$ is the Rayleigh range of the driving field. Finally, to compare the simulated spectrum with the attosecond spectrum isolated experimentally using a Zr filter, we apply a spectral filter corresponding to the transmission of a $200 \mathrm{~nm} \mathrm{Zr}$ foil taken from Ref. [4]. The driving pulse parameters are chosen to be $\tau=4.5 \mathrm{fs}, I_{0}=7.5 \times 10^{14} \mathrm{~W} / \mathrm{cm}^{2}$, and $\varphi_{\mathrm{CEP}}=\pi / 2$ in the simulation corresponding to the pulse duration, peak intensity, and carrier-envelope phase of the driving field respectively.

\section{REFERENCES}

1. Q. Zhang, K. Zhao, J. Li, M. Chini, Y. Cheng, Y. Wu, E. Cunningham, and Z. Chang, "Suppression of driving laser in high harmonic generation with a microchannel plate," Opt. Lett. 39, 3670-3673 (2014).

2. J. Gagnon, E. Goulielmakis, and V. S. Yakovlev, "The accurate frog characterization of attosecond pulses from streaking measurements," Appl. Phys. B 92, 25-32 (2008).

3. E. Goulielmakis, M. Schultze, M. Hofstetter, V. S. Yakovlev,
J. Gagnon, M. Uiberacker, A. L. Aquila, E. M. Gullikson, D. T. Attwood, R. Keinberger, F. Krausz, and U. Kleineberg, "Single-cycle nonlinear optics," Science 320, 1614-1617 (2008).

4. "The center for x-ray optics, x-ray database," http://henke.lbl. gov/optical_constants/.

5. M. Lewenstein, P. Balcou, M. Y. Ivanov, A. L'Hullier, and P. B. Corkum, "Theory of high-harmonic generation by lowfrequency laser fields," Phys. Rev. A. 49, 2117-2132 (1994).

6. Z. Chang, "Single attosecond pulse and xuv supercontinuum in the high-order harmonic plateau," Phys. Rev. A 70, 043802 (2004).

7. C. Altucci, V. Tosa, and R. Velotta, "Beyond the single-atom response in isolated attosecond-pulse generation," Phys. Rev. A. 75, 061401(R) (2007).

8. E. Priori, G. Cerullo, M. Nisoli, S. Stagira, S. D. Silvestri, P. Villoresi, L. Poletto, P. Ceccherini, C. Altucci, R. Bruzzese, and C. de Lisio, "Nonadiabatic three-dimensional model of high-order harmonic generation in the few-optical-cycle regime," Phys. Rev. A. 61, 063801 (2000). 